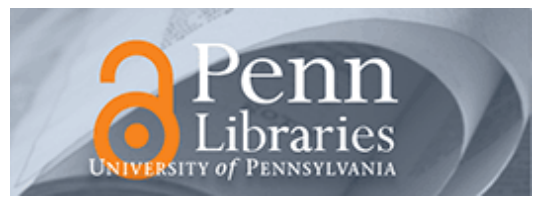

University of Pennsylvania

ScholarlyCommons

August 2007

\title{
Chemical and mechanical nerve root insults induce differential behavioral sensitivity and glial activation that are enhanced in combination
}

Sarah M. Rothman

University of Pennsylvania

Beth A. Winkelstein

University of Pennsylvania, winkelst@seas.upenn.edu

Follow this and additional works at: https://repository.upenn.edu/be_papers

\section{Recommended Citation}

Rothman, S. M., \& Winkelstein, B. A. (2007). Chemical and mechanical nerve root insults induce differential behavioral sensitivity and glial activation that are enhanced in combination. Retrieved from https://repository.upenn.edu/be_papers/96

Postprint version. Published in Brain Research, Volume 1181, November 2007, pages 30-43. Publisher URL: http://dx.doi.org/10.1016/j.brainres.2007.08.064

This paper is posted at ScholarlyCommons. https://repository.upenn.edu/be_papers/96

For more information, please contact repository@pobox.upenn.edu. 


\title{
Chemical and mechanical nerve root insults induce differential behavioral sensitivity and glial activation that are enhanced in combination
}

\begin{abstract}
Both chemical irritation and mechanical compression affect radicular pain from disc herniation. However, relative effects of these insults on pain symptoms are unclear. This study investigated chemical and mechanical contributions for painful cervical nerve root injury. Accordingly, the $C 7$ nerve root separately underwent chromic gut exposure, $10 \mathrm{gf}$ compression, or their combination. Mechanical allodynia was assessed, and glial reactivity in the $\mathrm{C} 7$ spinal cord tissue was assayed at days 1 and 7 by immunohistochemistry using GFAP and OX-42 as markers of astrocytes and microglia, respectively. Both chromic gut irritation and $10 \mathrm{gf}$ compression produced ipsilateral increases in allodynia over sham $(p<</ e m>0.048)$; combining the two insults significantly $(\mathrm{p}<</ \mathrm{em}>0.027)$ increased ipsilateral allodynia compared to either insult alone. Behavioral hypersensitivity was also produced in the contralateral forepaw for all injuries, but only the combined insult was significantly increased over sham $(p<</ e m>0.031)$. Astrocytic activation was significantly increased over normal $(p<</ e m>0.001)$ in the ipsilateral dorsal horn at 1 day after either compression or the combined injury. By day 7, GFAP-reactivity was further increased for the combined injury compared to day 1 ( $p<</ e m>0.001)$. In contrast, spinal OX-42 staining was generally variable, with only mild activation at day 1. By day 7 after the combined injury, there were significant $(\mathrm{p}<</ \mathrm{em}>0.003$ ) bilateral increases in $0 X-42$ staining over normal. Spinal astrocytic and microglial reactivity follow different patterns after chemical root irritation, compression, and a combined insult. The combination of transient compression and chemical irritation produces sustained bilateral hypersensitivity, sustained ipsilateral spinal astrocytic activation and late onset bilateral spinal microglial activation.
\end{abstract}

\section{Keywords}

radiculopathy, disc herniation, pain, glia, nerve root, spinal cord

\section{Comments}

Postprint version. Published in Brain Research, Volume 1181, November 2007, pages 30-43. Publisher URL: http://dx.doi.org/10.1016/j.brainres.2007.08.064 


\title{
Research Report
}

\section{Chemical and mechanical nerve root insults induce differential behavioral sensitivity and glial activation that are enhanced in combination}

\author{
Sarah M. Rothman ${ }^{a}$, Beth A. Winkelstein ${ }^{a, b, *}$ \\ aDepartment of Bioengineering, University of Pennsylvania, Philadelphia, PA 19104, USA \\ bepartment of Neurosurgery, University of Pennsylvania, Philadelphia, PA 19104, USA
}

\section{A R T I C L E I N F O}

Article history:

Accepted 28 August 2007

Keywords:

Radiculopathy

Disc herniation

Pain

Glia

Nerve root

Spinal cord

\begin{abstract}
A B S T R A C T
Both chemical irritation and mechanical compression affect radicular pain from disc herniation. However, relative effects of these insults on pain symptoms are unclear. This study investigated chemical and mechanical contributions for painful cervical nerve root injury. Accordingly, the C7 nerve root separately underwent chromic gut exposure, 10gf compression, or their combination. Mechanical allodynia was assessed, and glial reactivity in the $\mathrm{C} 7$ spinal cord tissue was assayed at days 1 and 7 by immunohistochemistry using GFAP and OX-42 as markers of astrocytes and microglia, respectively. Both chromic gut irritation and 10gf compression produced ipsilateral increases in allodynia over sham $(p<0.048)$; combining the two insults significantly $(p<0.027)$ increased ipsilateral allodynia compared to either insult alone. Behavioral hypersensitivity was also produced in the contralateral forepaw for all injuries, but only the combined insult was significantly increased over sham $(p<0.031)$. Astrocytic activation was significantly increased over normal $(p<0.001)$ in the ipsilateral dorsal horn at 1 day after either compression or the combined injury. By day 7, GFAP-reactivity was further increased for the combined injury compared to day $1(p<0.001)$. In contrast, spinal OX-42 staining was generally variable, with only mild activation at day 1 . By day 7 after the combined injury, there were significant $(p<0.003)$ bilateral increases in OX-42 staining over normal. Spinal astrocytic and microglial reactivity follow different patterns after chemical root irritation, compression, and a combined insult. The combination of transient compression and chemical irritation produces sustained bilateral hypersensitivity, sustained ipsilateral spinal astrocytic activation and late onset bilateral spinal microglial activation.
\end{abstract}

ㄷ 2007 Elsevier B.V. All rights reserved.

\section{Introduction}

Nearly one-third of Americans suffer from acute, chronic, or chronic intermittent pain; chronic pain affects as many as $1 / 2$ of the general population (Hasselstrom et al., 2002; Elliott et al., 1999). In particular, disc herniation with nerve root compression is the most common cause of radicular pain (Frymoyer, 1988; Hart et al., 1995; Ohnmeiss et al., 1997; Atlas et al., 2005).

\footnotetext{
* Corresponding author. Department of Bioengineering, University of Pennsylvania, 240 Skirkanich Hall, 210 S. 33rd Street, Philadelphia, PA 19104 6321, USA. Fax: +1 2155732071.

E-mail address: winkelst@seas.upenn.edu (B.A. Winkelstein).

URL: http://spinepain.seas.upenn.edu/ (B.A. Winkelstein).
}

0006-8993/\$ - see front matter @ 2007 Elsevier B.V. All rights reserved.

doi:10.1016/j.brainres.2007.08.064 
Low back pain and sciatica often result from disc herniation (Boos et al., 1995); cervical disc herniation, while not as common as lumbar disc herniation, is nonetheless a leading cause of cervical radiculopathy (Ellenberg et al., 1994). Both chemical and mechanical factors contribute to radicular pain from disc herniation; the inflammatory material of the disc comes in contact with the nerve root, causing impingement and compression (Wall and Melzak, 1994; Rydevik et al., 1996; Olmarker and Myers, 1998). Disc tissue, particularly the nucleus pulposus, initiates an inflammatory response and produces and releases pro-inflammatory cytokines (Weiler et al., 2004; Saal, 1995; Goupille et al., 1998; Murata et al., 2004; Mulleman et al., 2005; Yoshida et al., 2005). For these reasons, the common approach to clinical treatment for painful disc herniation is surgery to remove the inflammatory material and unload the root (Loupasis et al., 1999; DeLeo and Winkelstein, 2002).

Despite the common notion that removing a putative insult can alleviate pain, there remains uncertainty about whether mechanical or chemical factors contribute more to pain from a disc herniation. In fact, surgery to relieve pressure to nerve roots does not always provide pain relief (Loupasis et al., 1999; Mulleman et al., 2005). These surgical studies imply that either chronic radicular pain is mediated by a chemical component that is not detectable through conventional imaging, or that a transient compressive insult may induce persistent pain. Chemical mediators, transient compression, or their combination may have a mechanistic role in cervical nerve root pain.

Many pain models have been developed to mimic clinical lumbar disc herniation (McCarron et al., 1987; Olmarker and Myers, 1998; Hou et al., 2003; Murata et al., 2004, 2005). Either nucleus pulposus is expelled by a small injection of air, or excised and applied directly to the nerve root (Olmarker et al., 1993, 1995, 1997, 2002; Otani et al., 1997; Olmarker and Myers, 1998; Yabuki et al., 1998; Skouen et al., 1999; Kawakami et al., 2003; Cornefjord et al., 2004; Kallakuri et al., 2005; Onda et al., 2005). These models induce behavioral hypersensitivity, as measured by allodynia, (pain provoked by a normally non-painful stimulus) and hyperalgesia (an increased response to a painful stimulus). While autologous disc material may be a clinically relevant model of the local injury scenario, such approaches present logistical challenges and do not enable separation of the specific and relative effects of either the mechanical and/or chemical contributions of the insult. As such, lumbar radiculopathy models have also separated these injury components to investigate their effects on behavioral hypersensitivity (Olmarker et al., 1993; Maves et al., 1993; Kawakami et al., 1994a,b; Kajander et al., 1996; Colburn et al., 1999; Hashizume et al., 2000; Hou et al., 2003; Murata et al., 2004; Winkelstein and DeLeo, 2004; Hubbard and Winkelstein, 2005). Though radicular pain models implement ligation with chromic gut to simultaneously apply compression and chemical irritation in low back pain (Maves et al., 1993; Kawakami et al., 1994a,b; Kajander et al., 1996; Xu et al., 1996; Colburn et al., 1999; Hashizume et al., 2000; Winkelstein and DeLeo, 2004; Robinson and Meert, 2005), mechanistic information for cervical disc herniation is limited.

Spinal glia contribute to the onset and development of neuropathic and radiculopathic pain (Colburn et al., 1999; Hashizume et al., 2000; Winkelstein and DeLeo, 2002; Newman, 2003; Watkins and Maier, 2003; McMahon et al., 2005; DeLeo et al., 2006; Narita et al., 2006). Activation of spinal microglia, the resident macrophages of the central nervous system, occurs before astrocytic activation, and includes their transformation to a phagocytic state, increases in cell surface markers, and release of inflammatory mediators (Watkins and Maier, 2003; McMahon et al., 2005; DeLeo et al., 2006). Ligation with chromic gut suture elicits greater spinal microglial activation than silk suture, despite similar astrocytic activation for the two suture types (Hashizume et al., 2000). It remains to be seen whether spinal glial activation is produced following cervical nerve root chemical irritation alone, and if so, whether it is modulated with the addition of transient compression.

While many studies have utilized chromic gut suture to mimic disc herniation, no report to date has separated mechanically and chemically induced behavioral sensitivity; nor have these effects been studied for a transient compression. This study examined the hypothesis that exposure of the cervical nerve root to either a chemical stimulus (chromic gut suture material) or transient mechanical compression of the nerve root produces behavioral hypersensitivity and spinal glial activation that are increased when the two insults are combined. This hypothesis was tested by applying either transient compression or chromic gut directly to the cervical nerve root, or both insults simultaneously. Rats underwent separate procedures: cervical nerve root exposure to chromic gut suture material (chromic exposure), 10gf transient compression (10gf compression), chromic gut exposure with supplemental compression (chromic+10gf compression), or root exposure (sham). Mechanical allodynia was measured in bilateral forepaws and cervical spinal cord tissue was harvested on days 1 and 7 for immunohistochemical analysis to detect astrocytic and microglial reactivity in the bilateral dorsal horns at C7. Both qualitative and quantitative methods were used to assess glial activation in order to compare the findings to the literature (Colburn et al., 1999; Hubbard and Winkelstein, 2005) and to provide objective quantification of activation. The goal of this study was to identify the relative roles of chemical irritation and transient mechanics in mediating behavioral sensitivity from cervical nerve root injury and to provide insight into relationships between spinal glial activation and behavioral outcomes.

\section{2. $\quad$ Results}

\subsection{Behavioral hypersensitivity}

Ipsilateral mechanical allodynia was present following all types of insults: chromic exposure, $10 \mathrm{gf}$ compression and chromic $+10 \mathrm{gf}$ compression. Sham procedures did not produce increases in allodynia over baseline in either forepaw (Figs. 1 and 2). Exposure of the nerve root to chromic gut material produced an immediate, albeit slight, increase in mechanical allodynia in the ipsilateral forepaw (Fig. 1). This increase was significant over sham $(p<0.041)$ on day 1 for testing with the $2 \mathrm{~g}$ and $4 \mathrm{~g}$ filaments and on day 3 for testing with all filaments (Fig. 1). Yet, the increase in allodynia following chromic exposure was only transient, and by day 5 allodynia was not significantly different than sham (Fig. 1). Transient compression produced increases in mechanical allodynia in the ipsilateral forepaw that were sustained (Fig. 1). This increase was significant over sham at 


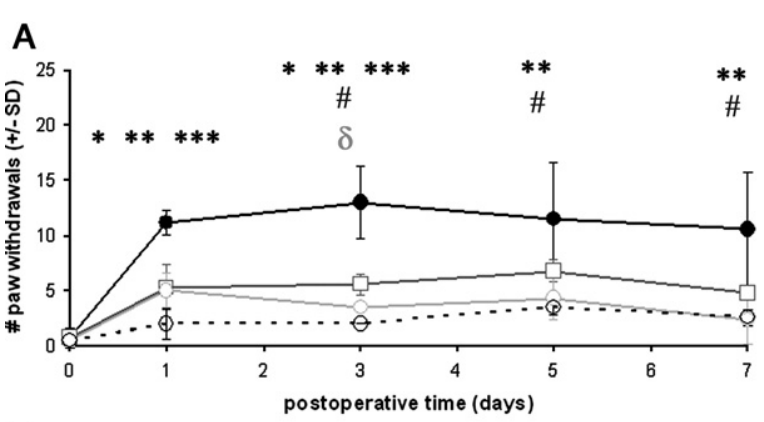

B
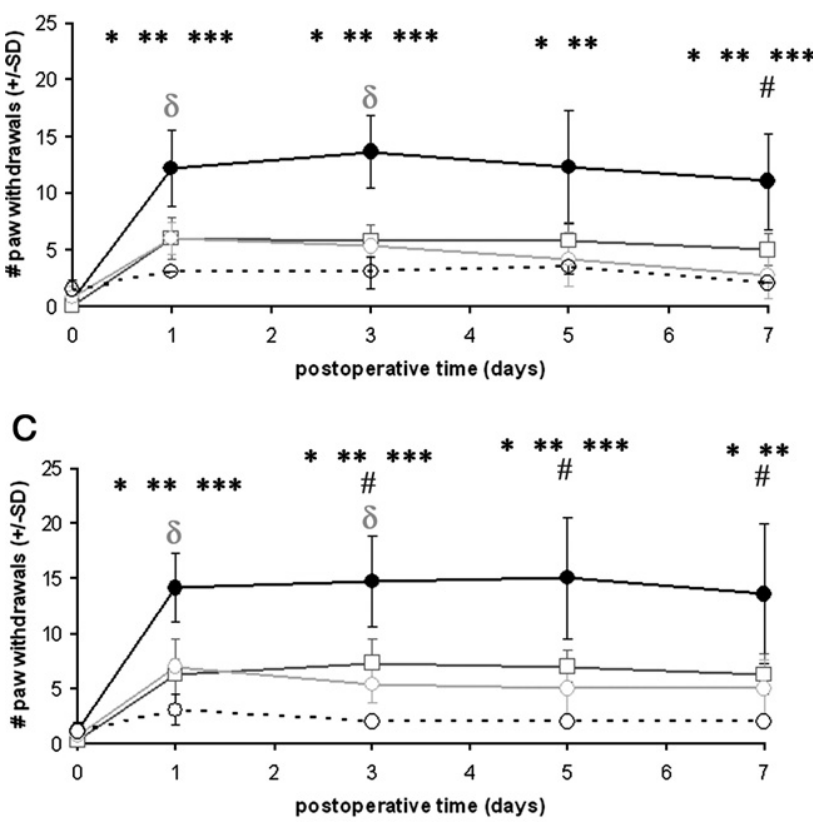

Fig. 1 - Average mechanical allodynia in the ipsilateral forepaw for sham ( $)$ ), chromic exposure ( ), 10gf compression ( $\square$ ) and chromic $+10 \mathrm{gf}$ compression $(\bullet)$. Allodynia is measured by the number of paw withdrawals for stimulation with each von Frey filament: (A) $1.4 \mathrm{~g}$, (B) $2 \mathrm{~g}$, and (C) $4 \mathrm{~g}$. Chromic exposure produced a slight increase in mechanical allodynia that was significant over sham $(p<0.041)$ on day 1 for testing with the $2 \mathrm{~g}$ and $4 \mathrm{~g}$ filaments and on day 3 for testing with all filaments. By day 5 chromic exposure was not significantly different than sham. Following $10 \mathrm{gf}$ compression, ipsilateral allodynia was sustained, displaying significant elevation over sham on day $7(p<0.047)$. Chromic $+10 \mathrm{gf}$ compression produced significant increases in ipsilateral allodynia over chromic exposure $(p<0.01), 10 \mathrm{gf}$ compression $(p<0.033)$ and sham $(p<0.043)$. Asterisks indicate significant difference between chromic $+10 \mathrm{gf}$ compression and sham (*), chromic exposure $\left({ }^{* *}\right)$ and $10 \mathrm{gf}$ compression $\left({ }^{* * *}\right)$. Pound sign $(")$ indicates significant difference between $10 \mathrm{gf}$ compression and sham, and delta $(\delta)$ indicates significant difference between chromic exposure and sham.

days 3 and 5 for testing with the $1.4 \mathrm{~g}$ and $4 \mathrm{~g}$ filaments and on day 7 for testing with all filaments $(p<0.047)$ (Fig. 1). No significant differences in mechanical allodynia were found between chromic exposure and $10 \mathrm{gf}$ compression. However, the combination of compression and chromic gut irritation together produced a robust increase in allodynia in the ipsilateral forepaw at all time points (Fig. 1). Allodynia for chromic $+10 \mathrm{gf}$ compression was significantly greater $(p<0.01)$ than that for chromic exposure at all time points with all filaments (Fig. 1). Allodynia for chromic $+10 \mathrm{gf}$ compression was also significantly greater than that for $10 \mathrm{gf}$ compression at days 1 and 3 for all filaments $(p<0.007)$, and at day 5 for testing with the $4 \mathrm{~g}$ filament $(p<0.027)$ and day 7 for testing with the $2 \mathrm{~g}$ filament $(p<0.033)$. Allodynia produced by the combination of insults was also significantly greater than sham at all time points for testing with the $2 \mathrm{~g}$ and $4 \mathrm{~g}$ filaments $(p<0.043)$, and at days 1 and 3 for testing with the $1.4 \mathrm{~g}$ filament $(p<0.001)$ (Fig. 1).

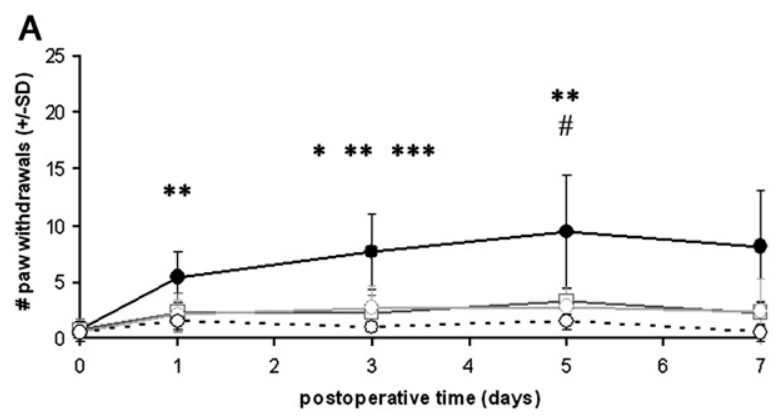

B

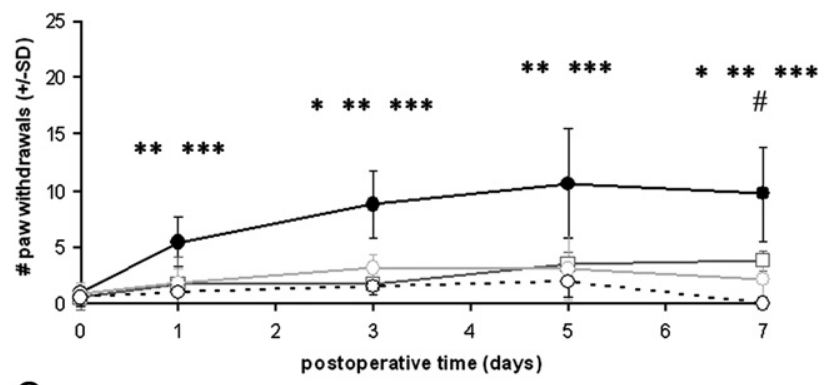

C

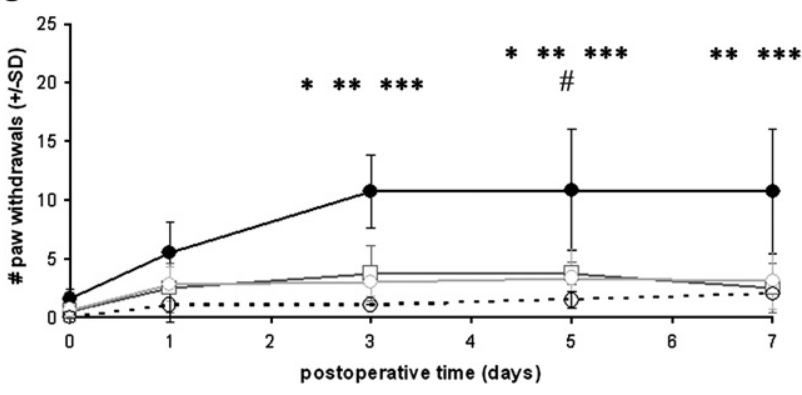

Fig. 2 - Average mechanical allodynia in the contralateral forepaw for sham (०), chromic exposure (), 10gf compression (口) and chromic $+10 \mathrm{gf}$ compression ( $\bullet$ ) for (A) $1.4 \mathrm{~g}$, (B) $2 \mathrm{~g}$, and (C) $4 \mathrm{~g}$ von Frey filaments. Chromic exposure and $10 \mathrm{gf}$ compression produced slight increases in allodynia over sham. However, chromic $+10 \mathrm{gf}$ compression produced increased contralateral allodynia compared to sham, chromic exposure and $10 \mathrm{gf}$ compression, for testing with the (A) $1.4 \mathrm{~g}$, (B) $2 \mathrm{~g}$, and (C) $4 \mathrm{~g}$ filaments. Asterisks indicate significant differences between chromic $+10 \mathrm{gf}$ compression and sham (*), chromic exposure (**) and 10gf compression (***). Pound sign (\#) indicates significant difference between $10 \mathrm{gf}$ compression and sham. 
Mechanical allodynia was produced in the contralateral forepaw of rats following chemical insult with the chromic gut or transient compression, despite being remote to the injury site. Contralateral mechanical allodynia was not as profound as that produced on the side ipsilateral to the nerve root injury and was not sustained (Figs. 1 and 2). Nonetheless, contralateral allodynia was slightly elevated over baseline and sham for both the chromic exposure and 10gf compression groups, and

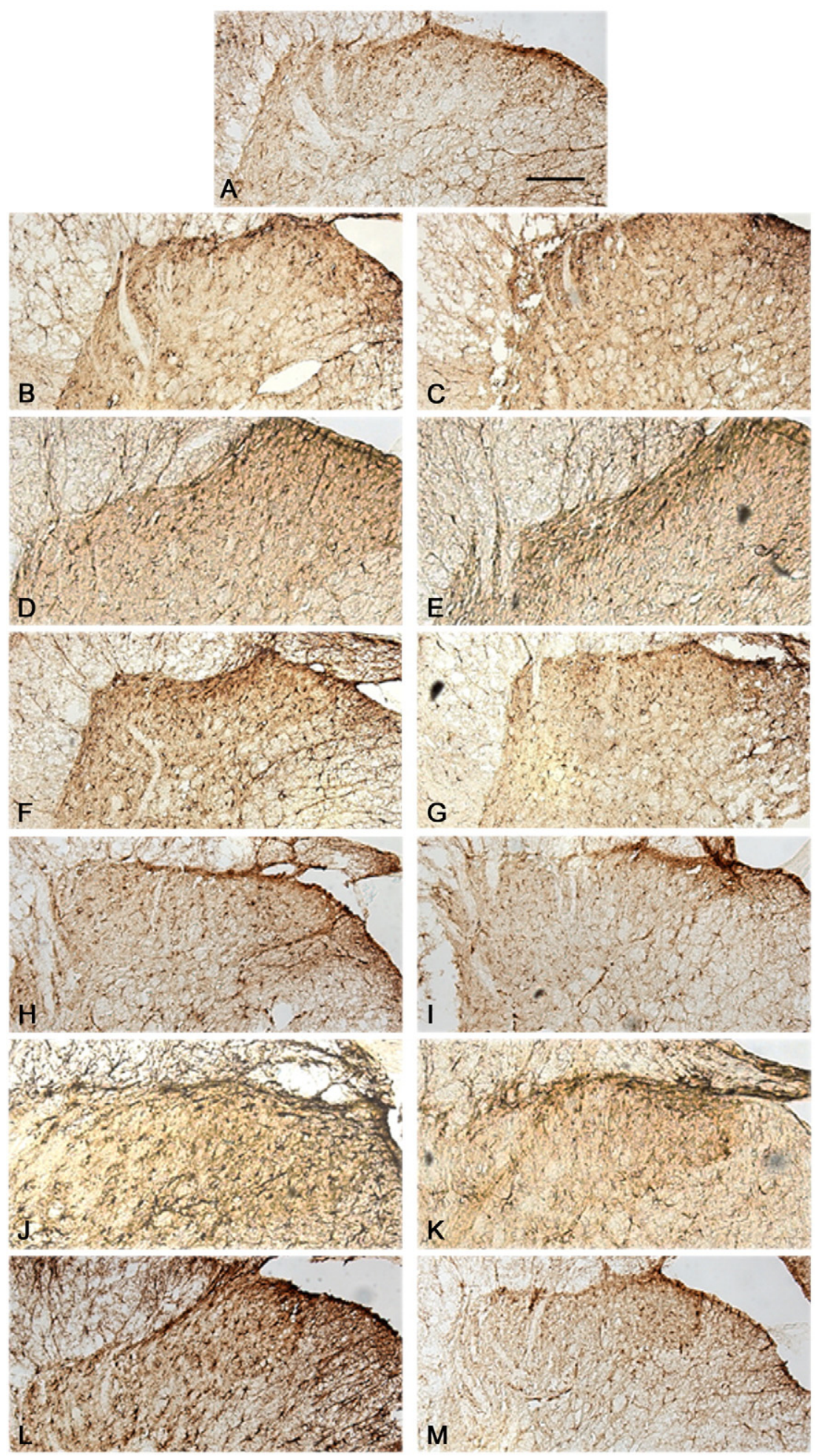

Fig. 3 - Representative ipsilateral (B, D, F, H, J, L) and contralateral (C, E, G, I, K, M) C7 spinal cord sections stained against GFAP after chromic exposure (B, C, H, I), 10gf compression (D, E, J, K) or chromic +10gf compression (F, G, L, M) on day 1 (B-G) or day 7 (H-M) following injury. A matching normal naive sample (A) was assigned baseline levels of staining. Scale bar shown in panel $A$ is $100 \mu \mathrm{m}$ and applies to all panels. 


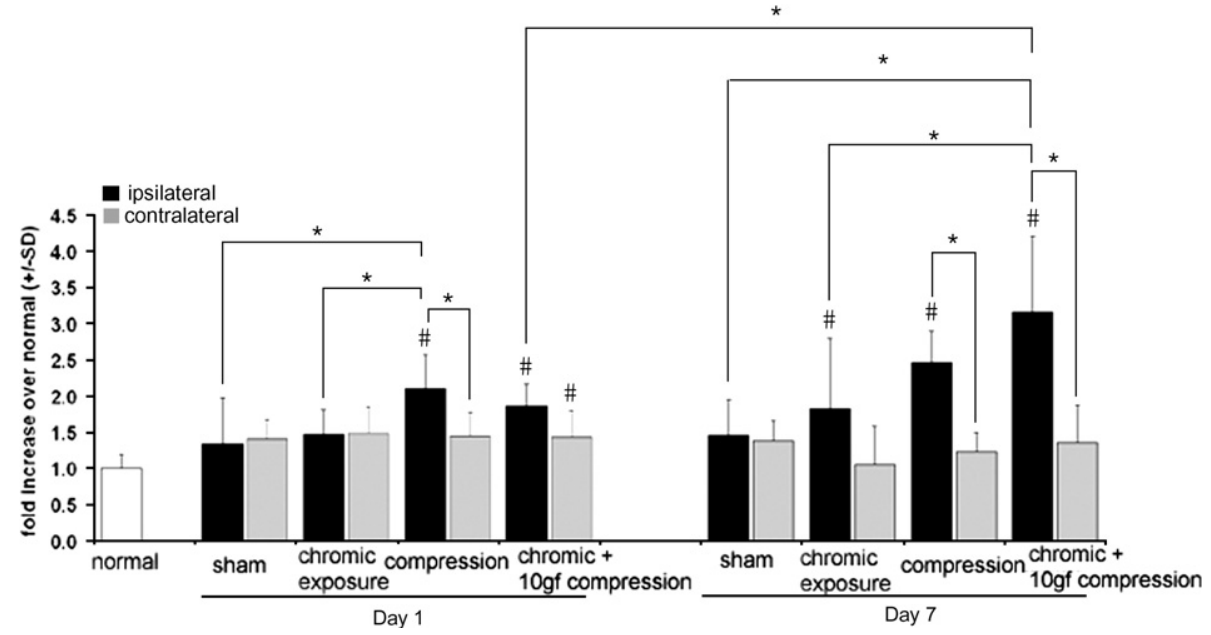

Fig. 4 - Automated densitometry results quantifying percentage of positive pixels reactive for GFAP staining, relative to normal levels at day 1 and day 7 following chromic exposure, 10gf compression, chromic $+10 \mathrm{gf}$ compression and sham. Significant $(" p<0.001)$ elevations over normal were detected for ipsilateral $10 \mathrm{gf}$ compression and chromic $+10 \mathrm{gf}$ compression at day 1 . Ipsilateral $10 \mathrm{gf}$ compression was also significantly elevated over sham, chromic exposure, and contralateral expression at day 1 (" $p<0.022)$. All injury groups were elevated in the ipsilateral dorsal horn over normal at day 7 ( $p<0.044)$ with both $10 \mathrm{gf}$ compression and chromic $+10 \mathrm{gf}$ compression also elevated over contralateral expression $\left({ }^{*} p<0.003\right)$. Ipsilateral GFAP staining at day 7 for chromic $+10 \mathrm{gf}$ compression was significantly greater than the expression at day 1 in the ipsilateral dorsal horn, the expression at day 7 following chromic exposure and the expression at day 7 following $\operatorname{sham}\left({ }^{*} p<0.001\right)$.

increased further with the combination of insults (Fig. 2). Chromic exposure produced only mild contralateral allodynia that was not significantly different than sham at any time point. Transient compression produced some significant increases over sham $(p<0.044)$ (Fig. 2), but these were inconsistent across von Frey filaments and not sustained. However, the combination of chromic exposure and transient compression increased contralateral allodynia compared to sham, chromic exposure and $10 \mathrm{gf}$ compression (Fig. 2). While the trend was similar to that of the ipsilateral forepaw, contralateral allodynia generally had a slower onset and lower responses (Figs. 1 and 2). A chromic $+10 \mathrm{gf}$ compression produced significant increases in contralateral allodynia over both chromic exposure and $10 \mathrm{gf}$ compression at days 3, 5, and 7, for testing with the $2 \mathrm{~g}$ and $4 \mathrm{~g}$ filaments $(p<0.039)$. These increases were also significant over sham $(p<0.026)$ at day 3 for all filaments (Fig. 2).

Table 1 - Immunohistochemical scoring of staining for GFAP reactivity

Nerve root insult Astrocytic (GFAP) staining

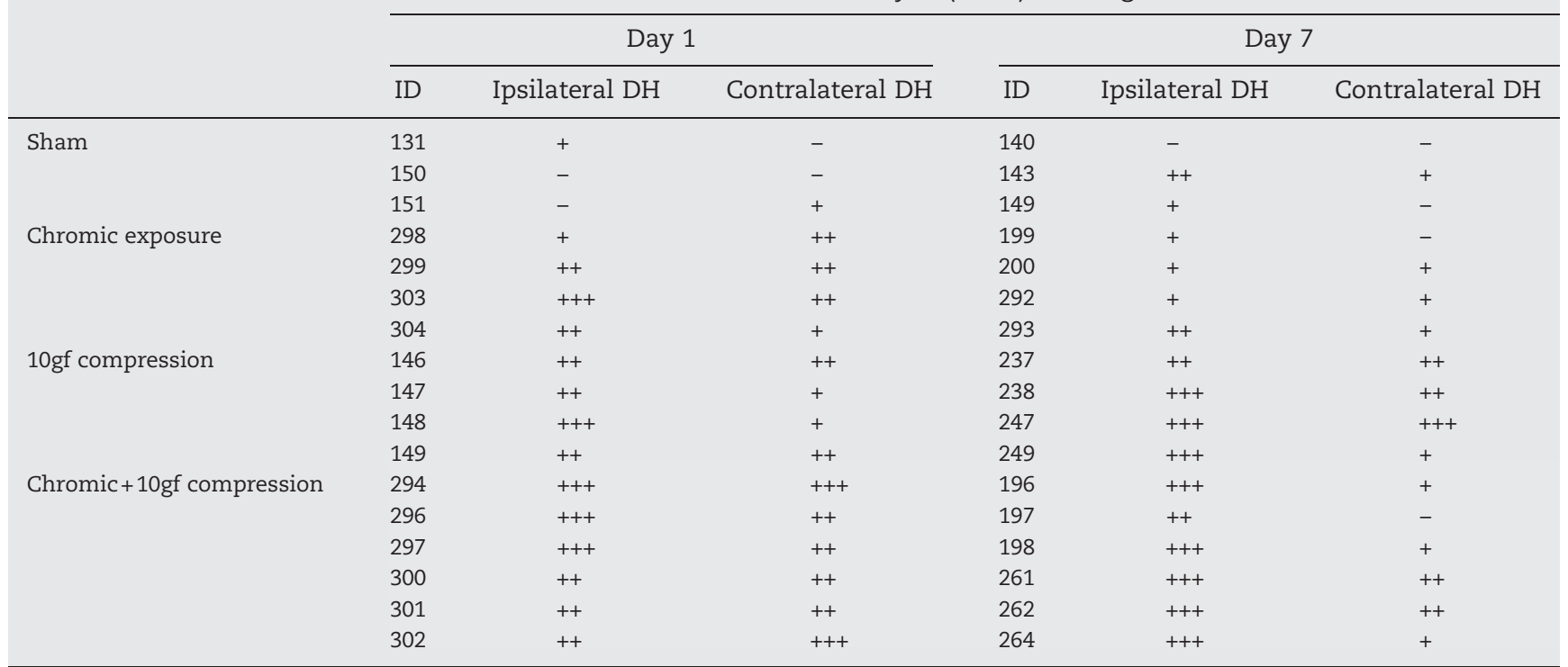

Assessments on a 4-point scale with the following gradients: (-) baseline staining, (+) mild response, (++) moderate response, and (+++) intense response.

ID - animal identification number; DH - dorsal horn. 


\subsection{Spinal glia}

Separate groups of rats were used for analysis of glial activation at days 1 and 7 following surgery. Tissue damage during harvest procedures precluded the use of spinal cord tissue from some rats in the behavioral study. Glial reactivity data at day 7 reflect only a subset of rats used for behavioral assays in each of the chromic exposure $(n=4)$ and chromic $+10 \mathrm{gf}$ compression $(n=6)$ groups.
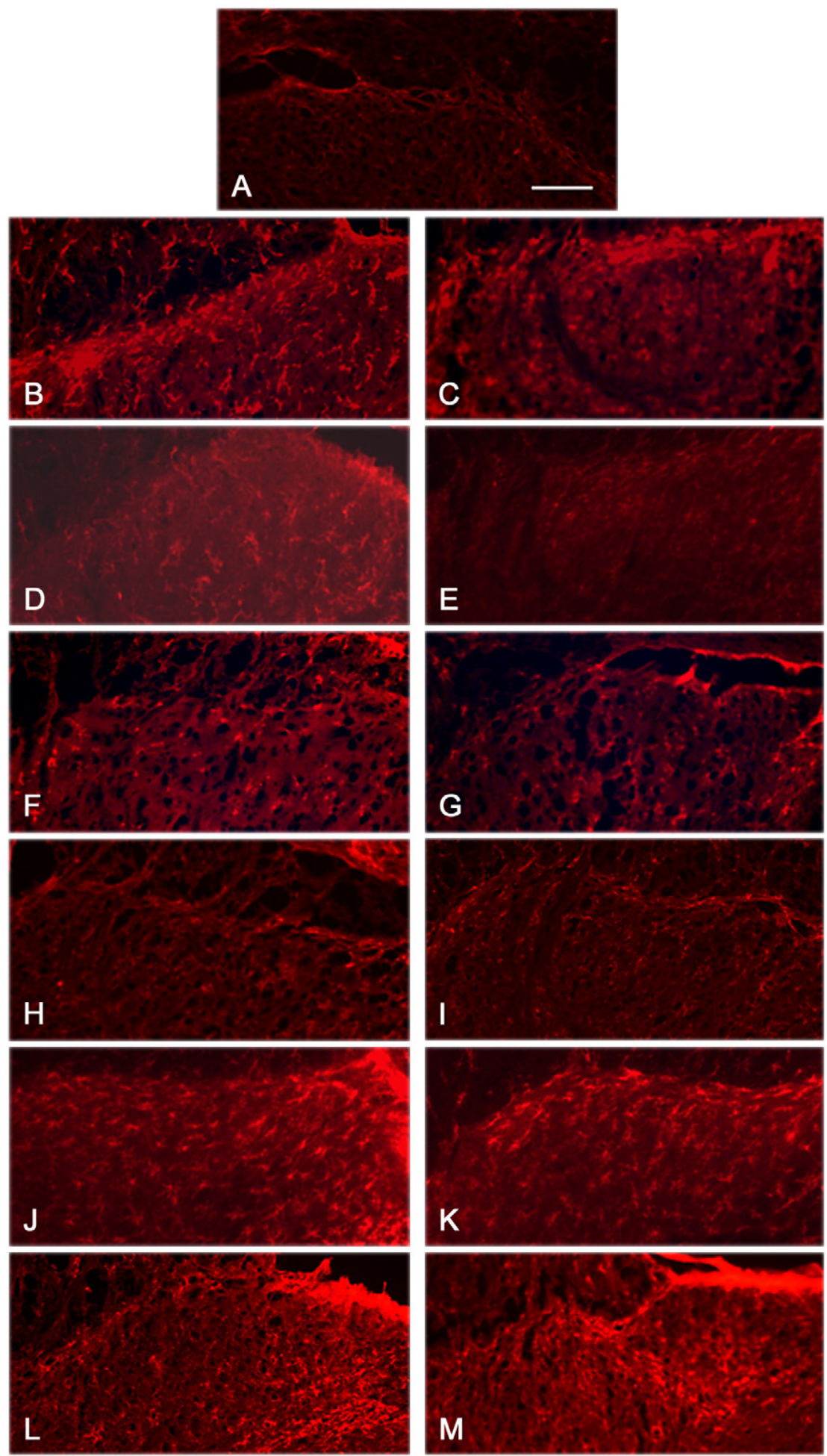

Fig. 5 - Representative ipsilateral (B, D, F, H, J, L) and contralateral (C, E, G, I, K, M) C7 spinal cord sections stained against OX-42 after chromic exposure (B, C, H, I), 10gf compression (D, E, J, K) or chromic +10gf compression (F, G, L, M) on day 1 (B-G) or day 7 (H-M) following injury. A matching normal naive sample (A) was assigned baseline levels of staining. Scale bar shown in panel $A$ is $100 \mu \mathrm{m}$ and applies to all panels. 


\subsection{Astrocytic activation}

GFAP staining for chromic exposure was inconsistent, while staining for $10 \mathrm{gf}$ compression was slightly more robust. The combination of insults increased GFAP staining over normal at day 1 and further at day 7 (Figs. 3 and 4; Table 1). Sham procedures produced only mild bilateral staining or no changes over normal, with only one subject displaying moderate $(++)$ staining and all others displaying baseline $(-)$ or mild $(+)$ staining (Table 1).

At day 1 following $10 \mathrm{gf}$ compression GFAP staining was increased compared to normal, with rats displaying moderate $(++)$ to intense $(+++)$ responses in the ipsilateral dorsal horn. This increase in ipsilateral GFAP staining was significant over normal $(p<0.001)$, as quantified by the densitometry analysis (Fig. 4). This increase was also significant compared to sham and chromic exposure $(p<0.022)$. However, increases in GFAP following $10 \mathrm{gf}$ compression were observed in the ipsilateral dorsal horn only, and were significant compared to the contralateral dorsal horn $(p=0.011)$, which displayed only mild-to-moderate reactivity (Fig. 4; Table 1). Ipsilateral increases in GFAP staining were maintained at day 7 following 10gf compression (Fig. 4); densitometry results indicate a significant increase in the ipsilateral dorsal horn over the contralateral dorsal horn and normal at day 7 ( $p<0.003)$. GFAP staining at day 7 following $10 \mathrm{gf}$ compression was slightly increased compared to day 1 , with rats displaying moderate or intense staining in the ipsilateral dorsal horn.

Chromic exposure produced irregular increases in ipsilateral GFAP staining at day 1 (Fig. 3; Table 1), but this was not significantly different from normal (Fig. 4). At day 7 after chromic exposure, ipsilateral staining increased and was significantly elevated over normal ( $p=0.044)$ (Fig. 4). Yet, this increase was slight and GFAP staining at day 7 following chromic exposure was significantly lower $(p<0.001)$ than GFAP reactivity produced at day 7 for chromic+10gf compression (Fig. 4).

Ipsilateral GFAP staining was robustly increased at day 1 for chromic $+10 \mathrm{gf}$ compression, with rats exhibiting moderate $(++)$ and intense $(+++)$ responses in both the ipsilateral and contralateral dorsal horns (Table 1). Both ipsilateral and contralateral increases in GFAP at day 1 were significant over normal $(p<0.046)$, as quantified by densitometry (Fig. 4). By day 7, ipsilateral GFAP staining following chromic $+10 \mathrm{gf}$ compression was further increased, with a significant elevation over day 1 expression $(p<0.001)$, and the chromic exposure $(p<0.001)$, sham $(p<0.001)$ and normal $(p<0.001)$ responses (Fig. 4). On day 7 , GFAP staining in the ipsilateral dorsal horn was significantly elevated over the contralateral dorsal horn $(p<0.001)$. Semiquantitative evaluation was graded as intense $(+++)$ in the ipsilateral dorsal horn at day 7 following chromic $+10 \mathrm{gf}$ compression for all but one rat, while contralateral staining was graded as baseline, mild or moderate for this same time point (Table 1).

\subsection{Microglial activation}

Both ipsilateral and contralateral OX-42 staining were highly variable between rats, with both chromic exposure and chromic + $10 \mathrm{gf}$ compression producing a range of scoring from baseline (-) to intense (+++) at each time point (Fig. 5; Table 2). Sham responses were consistently at normal levels, with only one subject at each time point displaying mild (+) responses (Table 2). Quantitative results were consistent with the qualitative assessments of variation in OX-42 staining for chromic exposure and chromic + $10 \mathrm{gf}$ compression; standard deviations in the densitometry measures were between $27 \%$ and $97 \%$ of their respective averages (Fig. 6). At day 1, no injury group displayed significant

Table 2 - Immunohistochemical scoring of staining for $0 X-42$ reactivity

Nerve root insult

Microglial (OX-42) staining

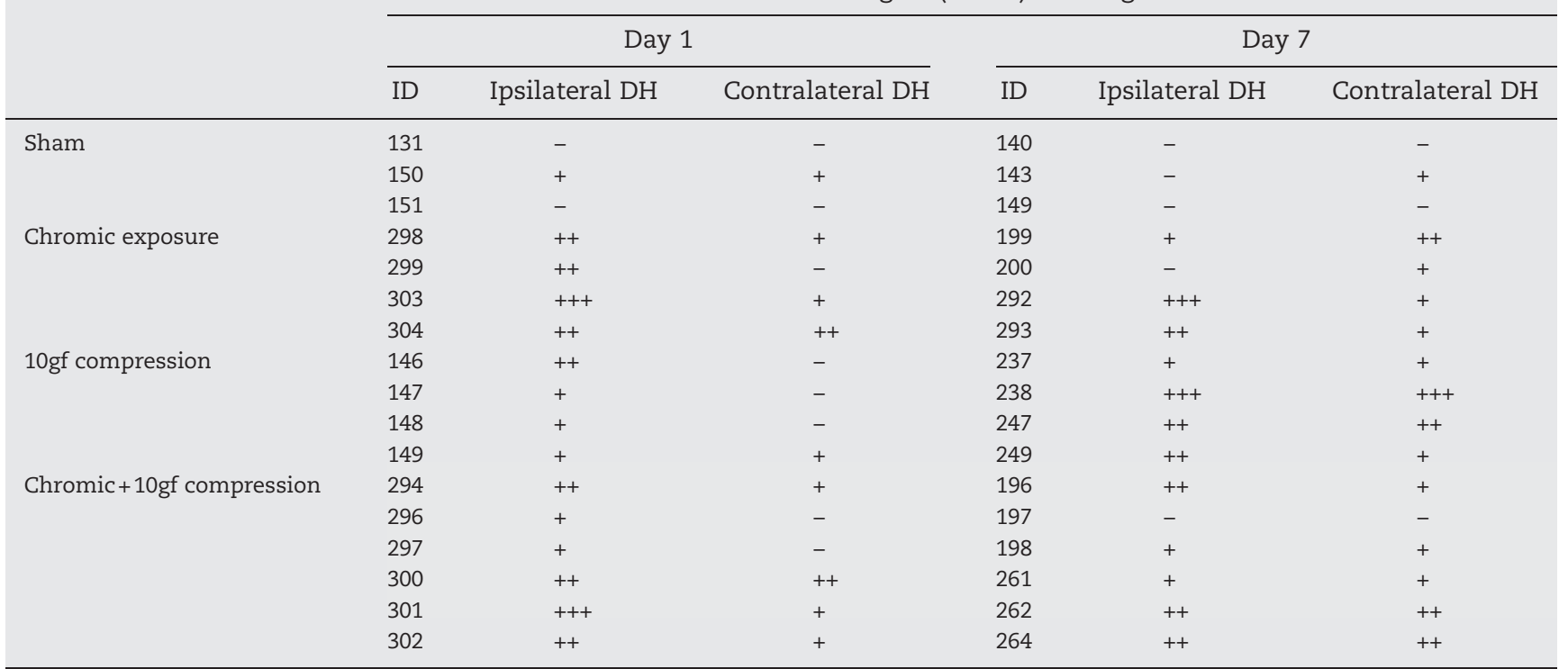

Assessments on a 4-point scale with the following gradients: (-) baseline staining, (+) mild response, (++) moderate response, and (+++) intense response.

ID - animal identification number; DH - dorsal horn. 


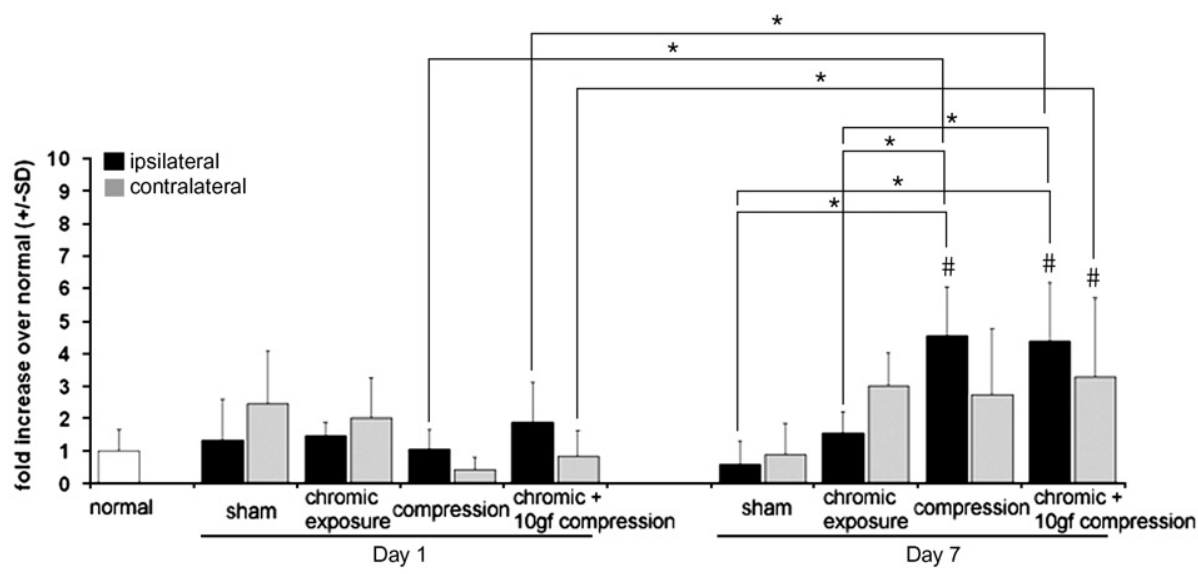

Fig. 6 - Automated densitometry results quantifying relative OX-42 staining at day 1 and day 7 following chromic exposure, 10gf compression, chromic $+10 \mathrm{gf}$ compression and sham. At day 1, reactivity in the bilateral dorsal horns for all injuries was not significantly different from sham or normal. By day 7, ipsilateral OX-42 staining following $10 \mathrm{gf}$ compression or chromic $+10 \mathrm{gf}$ compression was significantly increased compared to day 1 ( $\left.^{*} p<0.001\right)$. These day 7 increases were also significant compared to normal ( $\left.{ }^{p}<0.001\right)$, sham ( $\left.{ }^{*} p<0.001\right)$, and day 7 chromic exposure $\left({ }^{*} p<0.004\right)$. Contralateral increases were also significant compared to normal following chromic $+10 \mathrm{gf}$ compression $(" p=0.003)$ and day 1 chromic $+10 \mathrm{gf}$ compression $\left({ }^{*} p=0.008\right)$.

increases in ipsilateral OX-42 staining in the dorsal horn over sham or normal (Fig. 6).

By day 7, ipsilateral staining for chromic exposure remained generally unchanged compared to reactivity at day 1 (Fig. 6; Table 2). In contrast, at day 7 after $10 \mathrm{gf}$ compression, ipsilateral OX-42 reactivity was significantly increased compared to day 1 $(p<0.001)$. This ipsilateral increase between days 1 and 7 was also observed following chromic $+10 \mathrm{gf}$ compression $(p<0.001)$. Increases in ipsilateral OX-42 on day 7 following $10 \mathrm{gf}$ compression or chromic $+10 \mathrm{gf}$ compression were significant over chromic exposure $(p<0.004)$, sham $(p<0.001)$ and normal $(p<0.001)$ (Figs. 5 and 6). In addition, at this later time point, there was no significant difference in OX-42 responses detected between ipsilateral and contralateral dorsal horns for any injury type (Fig. 6). Further, at day 7 the chromic+10gf compression contralateral OX-42 responses were significantly elevated over normal $(p=0.003)$ and the contralateral spinal responses for that same injury at day $1(p=0.008)$ (Figs. 5 and 6$)$.

\section{Discussion}

Many studies have demonstrated a dependence of radicular pain on mechanical and chemical inputs (Kawakami et al., 1994a,b; Kajander et al., 1996; Olmarker et al., 1997; Hashizume et al., 2000; Olmarker et al., 2002; Hou et al., 2003; Winkelstein and DeLeo, 2004; Cornefjord et al., 2004; Hubbard and Winkelstein, 2005). This study is the first to our knowledge to examine such factors of chemical irritation and nerve root compression for transient loading to the cervical nerve roots. In particular, these results demonstrate a role for transient compression in mediating bilateral mechanical allodynia when combined with chemical factors. While allodynia was produced for chromic exposure, and also for $10 \mathrm{gf}$ compression, behavioral hypersensitivity was significantly increased and sustained when these injuries were combined (chromic $+10 \mathrm{gf}$ compression) (Figs. 1 and 2). Coupled with mechanical allody- nia, the combination of chemical irritation and transient compression of the nerve root also produced significant increases in both astrocytic and microglial reactivity in the dorsal horn of the spinal cord at day 7 (Figs. 4 and 6).

Although the combination of chromic gut exposure and transient compression induced robust increases in mechanical allodynia, chromic exposure alone also produced significantly elevated ipsilateral mechanical allodynia over sham at early time points. Allodynia following chromic exposure returned to sham levels by day 5 (Fig. 1), despite the fact that the chromic gut pieces remained in contact with the nerve root for the duration of the study. These results are in agreement with previous studies demonstrating slight and non-sustained increases in behavioral hypersensitivity following chromic exposure or a loose ligation of chromic suture around the lumbar nerve root (Lee et al., 2003; Winkelstein and DeLeo, 2004). Maves et al. (1993) demonstrated a 'dose response' for thermal sensitivity from sciatic nerve ligations using chromic gut suture; it is, therefore, likely that the amount or diameter of suture used in our study may not have been sufficient to induce a sustained behavioral effect. However, in studies using a chromic gut ligation it is difficult to separate the respective roles of the inflammatory and mechanical effects. The chromic gut suture can resorb and ligation with this material may induce further tightening as a consequence of tissue swelling, with both effects changing the local mechanics over time. Some studies of low back pain have utilized what those authors defined as a 'loose' ligation of the nerve root to place chromic suture in contact with the nerve root without applying additional pressure and a 'tight' ligation to apply mechanical compression in addition to the chemical irritation of the chromic suture (Kawakami et al., 1994a; Hashizume et al., 2000; Rutkowski et al., 2002). While described as 'loose' and 'tight' respectively, the mechanical conditions of the ligation groups were not explicitly defined by those authors. Yet, it is likely that the 'loose' chromic ligation did impose mechanical loading since gross pathology demonstrated narrowing in the nerve 
root following 'loose' ligations (Kawakami et al., 1994b; Hashizume et al., 2000). In our study, it is possible that the chromic gut pieces may have also imposed physical irritation to the nerve root, but this is very unlikely. The chromic pieces contacted the nerve root when gently placed along the root. They are very small and have little mass to provide any direct compression. In addition, visual inspection of the root at the time of tissue harvest showed no evidence of its narrowing or deformation in samples from the chromic exposure group.

The increased behavioral hypersensitivity for the combination of insults compared to a single insult (Figs. 1 and 2) is consistent with other reports of increases in mechanical and thermal hyperalgesia when these insults are combined compared to the application of disc material without compression of the root (Olmarker et al., 1997; Hou et al., 2003). Results presented here demonstrate that the combination of mechanical and chemical factors induces allodynia that is robustly increased compared to either chemical or mechanical injury alone (Figs. 1 and 2). Findings also suggest that a mechanical insult may be required for behavioral symptoms to be chronic, as chemical irritation alone does not produce sustained allodynia while the transient compression produce allodynia that is significant at day 7. Our data lend additional support to the hypothesis that the load threshold for producing persistent hypersensitivity in nerve root compression is lowered when chemical factors are present at injury (Winkelstein and DeLeo, 2004).

While bilateral allodynia has been previously reported for a unilateral insult (Tabo et al., 1999; Rutkowski et al., 2002; Araujo et al., 2003; Hou et al., 2003; Hubbard and Winkelstein, 2005), such mirror-image behavioral hypersensitivity was only significant for the combined mechanical and chemical insult (Fig. 2). This production of contralateral allodynia for a combined injury could imply that functional changes in the spinal cord are specifically induced in the interneurons that project to the nonoperated contralateral side only for specific injury paradigms and not for the chemical or mechanical injuries used in this study. Contralateral sensitivity has been previously reported for the combination of these insults, but not for exposure of the nerve root to chromic material or intervertebral disc samples (Maves et al., 1993; Rutkowski et al., 2002; Hou et al., 2003). Although contralateral allodynia has been induced by mechanical insults in the absence of any chemical loading, those studies implemented either ligation of multiple nerve roots or the spinal nerve, imposing a more severe sustained mechanical compression and one which may induce either a more robust, or continuous, barrage to the nociceptive cascades (Tabo et al., 1999; Araujo et al., 2003). While we did not specifically probe electrophysiologic properties of spinal interneurons, neuronal sensitization in the spinal cord can be initiated by increased neurotransmitter release, inflammatory cytokines, or directly by glial cells (Schiefer et al., 1999; Tabo et al., 1999; Cuellar et al., 2004; Inoue, 2005; Tsuda et al., 2005). Ipsilateral astrocytic activation was present at day 7 following compression and was further increased for the combined injury (Figs. 3 and 4; Table 1). Microglial activation at day 7 was also increased following compression, but was only on the side of injury; in contrast, for the addition of chromic suture, microglial reactivity at day 7 was observed bilaterally in the spinal cord (Figs. 5 and 6; Table 2). These increases in spinal glial activation following the com- bined injury at day 7 compared to either mechanical or chemical injuries alone imply that spinal inflammation is greatly increased when these factors are combined. Robust bilateral spinal inflammation, including increases in cell surface markers and inflammatory cytokines, and behavioral hypersensitivity have been reported for chromic ligation of lumbar nerve roots further supporting a role for inflammation in mediating spinal glial responses in painful nerve root injury (Rutkowski et al., 2002). Biochemical and functional immune changes in the spinal cord correlate with mechanical parameters for nerve root loading (i.e. applied loading rate, magnitude and duration); for example, imposed nerve root strain is significantly correlated with spinal mRNA levels for a host of inflammatory cytokines, implying sensitive biochemical responses to mechanical nerve root injury (Winkelstein et al., 2001a,b). Further, there is a direct relationship between root compression and neuronal function (Olmarker et al., 1989, 1990; Pedowitz et al., 1992; Mao et al., 1998; Yamaguchi et al., 1999). These results provide a host of candidate spinal responses that may be induced by injuries to the nerve root and are likely sensitive to the specific profile of the mechanical injury.

In the current study, the time course of activation of astrocytes and microglia was differentially modulated for the three types of injuries. At day 1, spinal astrocytic activation was evident in the ipsilateral horn for $10 \mathrm{gf}$ compression and chromic $+10 \mathrm{gf}$ compression, with an increase in activation on that side at day 7 following the combined injury (Fig. 4). However, microglial activation was not significantly elevated until day 7, and it was bilateral (Fig. 6). This is consistent with results from studies using silk ligations in which microglial activation was bilateral, whereas astrocytic activation was only apparent on the ipsilateral side of the spinal cord (Winkelstein and DeLeo, 2002). Bilateral increases in microglia, but not astrocytes, may be produced because microglia are more sensitive to ionic changes caused by neuronal sensitization which may occur on the contralateral side. Although little is known about what triggers glial activation, it has been shown that both microglia and astrocytes can be activated by depolarization of neighboring neurons, consistent with a tetrapartite model of the synapse (Kreutzberg, 1996; DeLeo et al., 2006). Yet, microglia may respond to these neuronal changes before astrocytes, and may be more sensitive to changes in neurotransmitters (Caggiano and Kraig, 1996). It is likely that in our combined injury microglia in the contralateral dorsal horn respond to small perturbations in extracellular ion homeostasis and this microglial activation then leads to further neuronal sensitization. Astrocytic activation may be present in the contralateral dorsal horn at a time point not probed in this study, as microglia produce and release molecules that can cause astrocytic activation (Kreutzberg, 1996; Newman, 2003; DeLeo et al., 2006). Studies of kinase activation and transcriptional changes demonstrate that spinal microglial activation can precede astrocytic activation (Tanga et al., 2004; Zhuang et al., 2005). While this study did probe the time course of glial activation, additional studies are needed of both acute and intermediate time points not studied here.

Differential spinal glial activation may also result from these cells having different thresholds for activation. Spinal astrocytic activation after ligation using silk is only slightly 
lower than that for a ligation with chromic gut (Hashizume et al., 2000; Winkelstein and DeLeo, 2002). This suggests that the initiation of spinal astrocytic reactivity may require a mechanical insult, and that it is only enhanced when this mechanical injury is accompanied by a chemical insult. In contrast, in those models, spinal microglial activation was greater for ligation with chromic suture relative to silk, suggesting that spinal microglia are responsive to chemical irritation. Microglial reactivity in our study increased bilaterally between days 1 and 7 for chromic+10gf compression, and unilaterally following compression alone (Fig. 6; Table 2), suggesting that microglia respond sensitively to the type and intensity of injury. Results presented here demonstrate only moderate microglial activation following chromic exposure (Fig. 6; Table 2) implying that it is the combination of insults, rather than simply a chemical insult alone, that causes robust microglial activation.

Our results, in the context of other studies, imply that microglia are more sensitive to chemical inputs than astrocytes are. This is in agreement with studies demonstrating that microglia respond to minor changes in their environment (Caggiano and Kraig, 1996; Kreutzberg, 1996). The apparent disparity in responses between microglia and astrocytes for different types of stimuli may be due to their varying roles in the central nervous system. Activated microglia release cytokines (TNF $\alpha$, IL-1 $\beta$, IL-6), which can then activate astrocytes, and also induces changes in neuronal excitability, as well as transcriptional changes in neurons that alter their sensitivity to stimuli (McMahon et al., 2005). The findings presented here are contradictory to this, with astrocytic reactivity significantly elevated over normal at day 1 following chromic $+10 \mathrm{gf}$ compression and microglia not exhibiting significant elevations until later at day 7 (Figs. 4 and 6). However, it is possible that the peak of microglial activation precedes the peak of astrocytic activation and occurs at an intermediate time point not probed in this study. In fact, following a painful lumbar nerve ligation, spinal extracellular signal-regulated protein kinases are activated in microglia with maximum activation on day 2, followed by a peak of pERK activation in astrocytes at day 21 (Zhuang et al., 2005). The bilateral microglial activation produced at day 7 for chromic $+10 \mathrm{gf}$ compression indicates prolonged microglial reactivity when chemical irritation is combined with transient compression in our model (Fig. 4). This could also suggest sustained cytokine release in a combined mechanical and chemical injury.

While our results demonstrate that the combination of putative insults causes robust and widespread behavioral and spinal glial responses (Figs. 1-6), additional studies directed at specific mechanical and chemical dose-effects are needed to fully define the relationship between injury parameters and pain. Although the present study used a repeatable, reliable chemical irritation as a discrete and uniform amount of chromic gut suture, the precise chemicals delivered and their effect on behavioral sensitivity were not specifically identified here. Quantitative studies specifically probing the role of spinal glia and their biochemical contributions, such as cytokine production, for different degrees of painful mechanical and/or chemical injury are necessary at additional time points. While the time points in this study were chosen as the onset and maintenance of behavioral hypersensitivity in this cervical radiculopathy model (Hubbard and Winkelstein, 2005; Rothman et al., 2005), it may be necessary to examine microglial activation at other time points. Further, it is recognized that qualitative microglial activation data showed a wide range of variability (Table 2) and associated densitometry results showed correspondingly highly standard deviations (Fig. 6); larger group sizes may also be necessary. While exposure of the nerve root to chromic gut suture does not directly mimic the clinical condition of nerve root irritation by the disc material, the chromic salts and pyrogallol that are used to produce chromic suture are known to be inflammatory to nervous tissue (Maves et al., 1993). Chromic suture, therefore, provides a basis for modeling the chemical component of painful disc herniation. In fact, chromic gut suture has been used extensively in models of low back pain to mimic the inflammatory effects of disc material (Kawakami et al., 1994a, b; Maves et al., 1993; Hashizume et al., 2000; Rutkowski et al., 2002; Lee et al., 2003; Winkelstein and DeLeo, 2004). These models also demonstrate a robust increase in behavioral hypersensitivity for chromic ligations (mechanical and chemical injury) compared to a mechanical ligation injury alone (Kim and Chung, 1992; Kawakami et al., 1994b; Hashizume et al., 2000), similar to the behavioral effects achieved by applying nucleus pulposus directly to the compressed nerve root (McCarron et al., 1987; Hou et al., 2003; Kawakami et al., 2003; Murata et al., 2004; Kallakuri et al., 2005). Further, behavioral outcomes from both chromic suture ligation and disc herniation models are similar to clinical symptoms from disc herniation, which show long-term sensitivity in the extremities (Ellenberg et al., 1994; Boos et al., 1995).

These results demonstrate that either chemical irritation or mechanical compression to the cervical nerve root can induce mechanical allodynia and spinal glial activation and that those responses are increased by the combination of these insults. Behavioral results from our study support that pain can be produced in the absence of a compression (Figs. 1 and 2). However, while chemical irritation alone may be responsible for some pain cases, a compressive event, even a transient one, can dramatically enhance behavioral sensitivity and such sensitivity may persist. Microglial activation was highly variable both between injury types and for individual rats in each injury group (Table 2); this may explain, in part, the individual variation in pain symptoms observed for humans. Studies are needed to specifically define the relationships between mechanical and chemical parameters and behavioral, cellular, and biochemical responses in quantitative approaches.

\section{Experimental procedure}

Experiments were performed using male Holtzman rats (Harlan Sprague-Dawley; Indianapolis, ID), weighing 250$350 \mathrm{~g}$ at the start of the study. Rats were housed under USDAand AAALAC-compliant conditions with a 12-h:12-h light/dark cycle and free access to food and water. All experimental procedures were approved by the University of Pennsylvania Institutional Animal Care and Use Committee and carried out according to the guidelines of the Committee for Research and Ethical Issues of the IASP (Zimmermann, 1983). 


\subsection{Surgical procedures}

All procedures were performed under inhalation anesthesia ( $4 \%$ halothane for induction, $2 \%$ for maintenance). Each rat received either nerve root exposure to chromic gut suture material (chromic exposure) $(n=10)$, transient nerve root compression (10gf compression) ( $n=8)$, chromic gut exposure with supplemental cervical nerve root compression (chromic $+10 \mathrm{gf}$ compression) $(n=13)$, or sham exposure (sham) $(n=6)$. For this study, previously described procedures of cervical dorsal root compression were used and modified to incorporate inflammatory components at the time of insult (Hubbard and Winkelstein, 2005; Rothman et al., 2005). Rats were placed in a prone position, and an incision was made in the skin from the base of the skull to the second thoracic vertebra. Muscle and soft tissue were cleared exposing the C6 and C7 laminae. A C6/C7 hemilaminectomy and partial facetectomy were performed on the right side to expose the spinal cord and right C7 dorsal nerve root. Procedures for the chromic exposure involved placing four pieces of 3-0 chromic gut suture (2 mm in length) (Surgical Specialties; Reading, PA) on the right C7 dorsal nerve root proximal to the dorsal root ganglia (DRG). Procedures for 10gf compression match those previously published (Hubbard and Winkelstein, 2005; Rothman et al., 2005). Briefly, a 10gf microvascular clip (World Precision Instruments, Inc) was applied to the right C7 dorsal nerve root proximal to the dorsal root ganglion. Compression was imposed for $15 \mathrm{~min}$, before clip removal. Procedures for chromic $+10 \mathrm{gf}$ compression involved those described for chromic exposure in addition to those described for $10 \mathrm{gf}$ compression. Compression was applied to the right $\mathrm{C} 7$ dorsal nerve root proximal to the DRG, removed after $15 \mathrm{~min}$, and then four chromic gut suture pieces were placed on the dorsal root as described above. Procedures for sham involved nerve root exposure only, as previously published (Rothman et al., 2005). Following surgery, all wounds were closed using 3-0 polyester suture and surgical staples. Rats were recovered in room air and monitored continuously.

\subsection{Behavioral assessment}

Following surgery, a subset of rats ( $n=6$ chromic exposure, $n=4$ $10 \mathrm{gf}$ compression, $n=7$ chromic $+10 \mathrm{gf}$ compression, $n=3$ sham) was evaluated for behavioral hypersensitivity after injury. Mechanical allodynia was measured in the ipsilateral and contralateral forepaws on each of postoperative days 1, 3, 5, and 7. Methods for quantifying forepaw allodynia have been previously validated for cervical nerve root injury (Hubbard and Winkelstein, 2005; Rothman et al., 2005). Prior to surgery, rats were acclimated to the tester and environment, and baseline measurements were recorded for 2 days. For each session, after $20 \mathrm{~min}$ of acclimation, rats were stimulated on the plantar surface of the ipsilateral and contralateral forepaws, separately, using three von Frey filaments (1.4, 2, 4 g) (Stoelting Co.; Wood Dale, IL). Each testing session consisted of three rounds of 10 stimulations each to each forepaw, separated by $10 \mathrm{~min}$. For each session with a given filament, the total number of withdrawals was counted for each paw and averages for each injury group were determined. A single tester performed all behavioral testing blinded to surgical procedures.

\subsection{Immunohistochemistry procedures}

In order to assess the temporal pattern of glial activation in the dorsal horn, C7 spinal cord was assayed for astrocytic and microglial activation using antibodies to glial fibrillary acidic protein (GFAP) and CR3/CD11b (OX-42), respectively. Cervical spinal cord tissue was harvested on each of days 1 and 7 following injury. Spinal cord tissue evaluated at day 1 was taken from a separate set of rats than that used for allodynia assessments ( $n=4$ chromic exposure, $n=410 \mathrm{gf}$ compression, $n=6$ chromic +10 gf compression, $n=3$ sham). For tissue harvest, rats were deeply anesthetized followed by transcardiac perfusion with $200 \mathrm{ml}$ of phosphate-buffered saline (PBS) and $300 \mathrm{ml}$ of $4 \%$ paraformaldehyde in PBS ( $\mathrm{pH} 7.4$ ). Following perfusion, the cervical spinal cord was exposed by laminectomy, the C7 segment of the cervical cord was harvested, and tissue was post-fixed in the $4 \%$ paraformaldehyde solution for $20 \mathrm{~min}$ (Hubbard and Winkelstein, 2005). Tissue was transferred to $30 \%$ sucrose/PBS and stored for 3 days at $4{ }^{\circ} \mathrm{C}$. Samples were freeze-mounted with OCT medium (Triangle Biomedical Sciences; Durham, NC) for axial cryosectioning. Matched spinal cord tissue from a naïve (un-operated) rat was also processed for normalization and comparison.

Four serial C7 spinal cord sections $(20 \mu \mathrm{m})$ from each rat were prepared for free-floating immunohistochemical staining. A polyclonal antibody to GFAP (Dako; Carpinteria, CA) was used as a marker of activated astrocytes. Slices were blocked with normal goat serum (Vector Labs; Burlingame, CA) for 20 min followed by incubation in a primary antibody directed against GFAP $(1: 20,000)$ overnight. Sections were then treated with a biotinylated goat anti-rabbit IgG, quenched in $0.3 \%$ peroxide solution, and developed using 3,3-diaminobenzidine (Vector Labs; Burlingame, CA). For a second set of matched axial sections, a monoclonal antibody to CR3/CD11b (OX-42; BD Pharmingen; San Diego, CA) was used as a marker of activated microglia. Spinal cord slices were blocked with normal goat serum (Vector Labs; Burlingame, CA) for $20 \mathrm{~min}$ and incubated overnight in an antibody directed against OX-42 (1:500). An Alexa546-conjugated goat anti-mouse secondary antibody (1:500) was used for immunofluorescent detection (Invitrogen; Carlsbad, CA). Previous studies were performed to determine optimal antibody dilutions. A negative control with no primary antibody staining was always included for verification of specificity of immunohistochemical techniques.

Two techniques were used to analyze glial activation. Semiquantitative scoring was used to grade the glial activation in each of the ipsilateral and contralateral dorsal horns to enable comparison to normal sections and results in the literature. In addition, automated densitometry was also used to quantify staining to enable quantitative and statistical comparisons. For each glial marker, two representative cord sections from each rat were photographed at $100 \times$ magnification using a digital camera and stereomicroscope system and Axiovision software (Zeiss; Thornwood, NY). For both analysis methods, the ipsilateral and contralateral dorsal horns relative to the injury site were assessed. For the semi-quantitative grading, two observers, blinded to the injury group graded the degree of activation. Separate scores were given to ipsilateral and contralateral sides. Grading was performed using an established 4-point scale, based on cell numbers, compactness, and intensity of staining (Colburn 
et al., 1999; Winkelstein and DeLeo, 2002; Hubbard and Winkelstein, 2005). Assessments were made using the following levels of gradation: baseline staining $(-)$, mild response $(+)$, moderate response (++), and intense response $(+++)$ (Colburn et al., 1999). Results from the first observer were verified by the second observer, and tabulated. For automated densitometry analysis, the same sections were used for semi-quantitative scoring. Images were cropped $(800 \times 200$ pixels $)$ to include the superficial lamina I-III only; fluorescent images were also inverted. Images were analyzed for percentage of pixels above a defined threshold (Abbadie et al., 1996). Threshold pixel intensity was chosen based on staining in normal tissue. This approach gives a quantitative measure of the degree and amount of staining above what is present in normal naïve tissue. Values were normalized, averaged and compared.

\subsection{Statistical analysis}

Significant differences in allodynia between groups were detected by a one-way ANOVA at each time point (SYSTAT; Richmond, CA). For quantitative densitometry, a one-way ANOVA was performed to compare differences over time (day 1, day 7), between injury groups (chromic exposure, 10gf compression, chromic + $10 \mathrm{gf}$ compression, sham), and between sides of the spinal cord (ipsilateral, contralateral). Significance was defined at $p<0.05$.

\section{Acknowledgments}

The authors thank Dr. Steven B. Nicoll for use of his microscope and imaging system and Raymond D. Hubbard for assessment of glial activation.

This work was funded by grant support from NIH (NIAMS) AR047564-02 and the Catharine D. Sharpe Foundation.

\section{REFEREN C ES}

Abbadie, C., Brown, J.L., Mantyh, P.W., Basbaum, A.I., 1996. Spinal cord substance P receptor immunoreactivity increases in both inflammatory and nerve injury models of persistent pain. Neuroscience 70, 201-209.

Araujo, M.C., Sinnott, C.J., Strichartz, G.R., 2003. Multiple phases of relief from experimental mechanical allodynia by systemic lidocaine: responses to early and late infusions. Pain 103, 21-29.

Atlas, S.J., Keller, R.B., Wu, Y.A., Deyo, R.A., Singer, D.E., 2005. Long-term outcomes of surgical and nonsurgical management of sciatica secondary to a lumbar disc herniation: 10 year results from the Maine lumbar spine study. Spine 30, 927-935.

Boos, N., Rieder, R., Schade, V., Spratt, K.F., Semmer, N., Aebi, M., 1995. 1995 Volvo award in clinical sciences: the diagnostic accuracy of magnetic resonance imaging, work perception, and psychosocial factors in identifying symptomatic disc herniations. Spine 20, 2613-2625.

Caggiano, A.O., Kraig, R.P., 1996. Eicosanoids and nitric oxide influence induction of reactive gliosis from spreading depression in microglia but not astrocytes.

J. Comp. Neurol. 369, 93-108.

Colburn, R.W., Rickman, A.J., DeLeo, J.A., 1999. The effect of site and type of nerve injury on spinal glial activation and neuropathic pain behavior. Exp. Neurol. 157, 289-304.
Cornefjord, M., Nyberg, F., Rosengren, L., Brisby, H., 2004. Cerebrospinal fluid biomarkers in experimental spinal nerve root injury. Spine 29, 1862-1868.

Cuellar, J.M., Montesano, P.X., Carstens, E., 2004. Role of TNF-alpha in sensitization of nociceptive dorsal horn neurons induced by application of nucleus pulposus to L5 dorsal root ganglion in rats. Pain 110, 578-587.

DeLeo, J.A., Winkelstein, B.A., 2002. Physiology of chronic spinal pain syndromes: from animal models to biomechanics. Spine 27, 2526-2537.

DeLeo, J.A., Tawfik, V.L., LaCroix-Fralish, M.L., 2006. The tetrapartite synapse: path to CNS sensitization and chronic pain. Pain 122, 17-21.

Ellenberg, M.R., Honet, J.C., Treanor, W.J., 1994. Cervical radiculopathy. Arch. Phys. Med. Rehabil. 75, 342-352.

Elliott, A., Smith, B., Penny, K., Smith, W., Chambers, W., 1999. The epidemiology of chronic pain in the community. Lancet 354, 1248-1252.

Frymoyer, J.W., 1988. Back pain and sciatica. N. Engl. J. Med. 318, 291-300.

Goupille, P., Jayson, M., Valat, J.P., Freemont, A.J., 1998. The role of inflammation in disk herniation-associated radiculopathy. Semin. Arthritis Rheum. 28, 60-71.

Hart, L.G., Deyo, R.A., Cherkin, D.C., 1995. Physician office visits for low back pain. Frequency, clinical evaluation, and treatment patterns from a U.S. national survey. Spine 20, 11-19.

Hashizume, H., DeLeo, J.A., Colburn, R.W., Weinstein, J.N., 2000. Spinal glial activation and cytokine expression after lumbar root injury in the rat. Spine 25, 1206-1217.

Hasselstrom, J., Liu-Palmgren, J., Rasjo-Wraak, G., 2002. Prevalence of pain in general practice. Eur. J. Pain 6, 375-385.

Hou, S.X., Tang, J.G., Chen, H.S., Chen, J., 2003. Chronic inflammation and compression of the dorsal root contribute to sciatica induced by the intervertebral disc herniation in rats. Pain 105, 255-264.

Hubbard, R.D., Winkelstein, B.A., 2005. Transient cervical nerve root compression in the rat induces bilateral forepaw allodynia and spinal glial activation: mechanical factors in painful neck injuries. Spine 30, 1924-1932.

Inoue, K., 2005. The function of microglia through purinergic receptors: neuropathic pain and cytokine release. Pharmacol. Ther. 109, 210-226.

Kajander, K.C., Pollock, C.H., Berg, H., 1996. Evaluation of hindpaw position in rats during chronic constriction injury (CCI) produced with different suture materials. Somatosens. Mot. Res. 13, 95-101.

Kallakuri, S., Takebayashi, T., Ozaktay, A.C., Chen, C., Yang, S., Wooley, P.H., Cavanaugh, J.M., 2005. The effects of epidural application of allografted nucleus pulposus in rats on cytokine expression, limb withdrawal and nerve root discharge. Eur. Spine J. 14, 956-964.

Kawakami, M., Weinstein, J.N., Spratt, K.F., Chatani, K., Traub, R., Meller, S.T., Gebhart, G.F., 1994a. Experimental lumbar radiculopathy: immunohistochemical and quantitative demonstrations of pain induced by lumbar root irritation of the rat. Spine 19, 1780-1794.

Kawakami, M., Weinstein, J.N., Chatani, K., Spratt, K.F., Meller, S.T., Gebhart, G.F., 1994b. Experimental lumbar radiculopathy: behavioral and histologic changes in a model of radicular pain after spinal nerve root irritation with chromic gut ligatures in the rat. Spine 19, 1795-1802.

Kawakami, M., Hashizume, H., Nishi, H., Matsumoto, T., Tamaki, T., Kuribayashi, K., 2003. Comparison of neuropathic pain induced by the application of normal and mechanically compressed nucleus pulposus to lumbar nerve roots in the rat. J. Orthop. Res. 21, 535-539.

Kim, S.H., Chung, J.M., 1992. An experimental model for peripheral neuropathy produced by segmental spinal nerve ligation in the rat. Pain 50, 355-363. 
Kreutzberg, G., 1996. Microglia: a sensor for pathological events in the CNS. Trends Neurosci. 19, 312-318.

Lee, D.H., Iyengar, S., Lodge, D., 2003. The role of uninjured nerve in spinal nerve ligated rats points to an improved animal model of neuropathic pain. Eur. J. Pain 7, 473-479.

Loupasis, G.A., Stamos, K., Katonis, P.G., Sapakis, G., Korres, D.S., Hartofilakidis, G., 1999. Seven- to 20-year outcome of lumbar disectomy. Spine 24, 2313-2317.

Mao, G.P., Konno, S., Arai, I., Olmarker, K., Kikuchi, S., 1998. Chronic double-level cauda equina compression: an experimental study on the dog cauda equina with analyses of nerve conduction velocity. Spine 23, 1641-1644

Maves, T.J., Pechman, P.S., Gebhart, G.F., Meller, S.T., 1993. Possible chemical contribution from chromic gut sutures produces disorders of pain sensation like those seen in man. Pain 54, 57-69.

McCarron, R.F., Wimpee, M.W., Hudkins, P.G., Laros, G.S., 1987. The inflammatory effect of nucleus pulposus. A possible element in the pathogenesis of low-back pain. Spine 12, 760-764.

McMahon, S.B., Cafferty, W.B.J., Marchand, F., 2005. Immune and glial cell factors as pain mediators and modulators. Exp. Neurol. 192, 444-462.

Mulleman, D., Mammou, S., Griffoul, I., Watier, H., Goupille, P., 2005. Pathophysiology of disk-related sciatica: I. Evidence supporting a chemical component. Jt. Bone Spine 73, 151-158.

Murata, Y., Onda, A., Rydevik, B., Takahashi, K., Olmarker, K., 2004. Distribution and appearance of tumor necrosis factor- $\alpha$ in the dorsal root ganglion exposed to experimental disc herniation in rats. Spine 29, 2235-2241.

Murata, Y., Olmarker, K., Takahashi, I., Takahashi, K., Rydevik, B., 2005. Effects of selective tumor necrosis factor-alpha inhibition to pain-behavioral changes caused by nucleus pulposus-induced damage to the spinal nerve in rats. Neurosci. Lett. 382, 148-152.

Narita, M., Yoshida, T., Nakajima, M., Narita, M., Miyatake, M., Takagi, T., Yajima, Y., Suzuki, T., 2006. Direct evidence for spinal cord microglia in the development of a neuropathic pain-like state in mice. J. Neurochem. 971, 1337-1348.

Newman, E.A., 2003. New roles for astrocytes: regulation of synaptic transmission. Trends Neurosci. 26, 536-542.

Ohnmeiss, D.D., Vanharanta, H., Ekholm, J., 1997. Degree of disc disruption and lower extremity pain. Spine 22, 1600-1605.

Olmarker, K., Myers, R.R., 1998. Pathogenesis of sciatic pain: role of herniated nucleus pulposus and deformation of spinal nerve root and dorsal root ganglion. Pain 78, 99-105.

Olmarker, K., Rydevik, B., Holm, S., 1989. Edema formation in spinal nerve roots induced by experimental, graded compression. An experimental study on the pig cauda equina with special reference to differences in effects between rapid and slow onset of compression. Spine 14, 569-573.

Olmarker, K., Holm, S., Rydevik, B., 1990. Importance of compression onset rate for the degree of impairment of impulse propagation in experimental compression injury of the procine cauda equina. Spine 15, 416-419.

Olmarker, K., Rydevik, B., Nordborg, C., 1993. Autologous nucleus pulposus induces neurophysiologic and histologic changes in porcine cauda equina nerve roots. Spine 18, 1425-1432.

Olmarker, K., Blomquist, M., Stromberg, J., Nannmark, U., Thomsen, P., Rydevik, B., 1995. Inflammatogenic properties of nucleus pulposus. Spine 20, 665-669.

Olmarker, K., Brisby, H., Yabuki, S., Nordborg, C., Rydevik, B., 1997. The effects of normal, frozen, and hyaluronidase-digested nucleus pulposus on nerve root structure and function. Spine 22, 471-475.

Olmarker, K., Storkson, R., Berge, O.G., 2002. Pathogenesis of sciatic pain. A study of spontaneous behavior in rats exposed to experimental disc herniation. Spine 27, 1312-1317.

Onda, A., Murata, Y., Rydevik, B., Larsson, K., Kikuchi, S., Olmarker, K., 2005. Nerve growth factor content in dorsal root ganglion as related to changes in pain behavior in a rat model of experimental lumbar disc herniation. Spine 30, 188-193.

Otani, K., Itaru, A., Mao, G.P., Konno, S., Olmarker, K., Kikuchi, S., 1997. Experimental disc herniation: evaluation of the natural course. Spine 22, 2894-2899.

Pedowitz, R.A., Garfin, S.R., Massie, J.B., Hargens, A.R., Swenson, M.R., Myers, R.R., Rydevik, B.L., 1992. Effects of magnitude and duration of compression on spinal nerve root conduction. Spine 17, 194-199.

Robinson, I., Meert, T.F., 2005. Stability of neuropathic pain symptoms in partial sciatic nerve ligation in rats is affected by suture material. Neurosci. Lett. 373, 125-129.

Rothman, S.M., Kreider, R.A., Winkelstein, B.A., 2005. Spinal neuropeptide responses in persistent and transient pain following cervical nerve root injury. Spine 30, 2491-2496.

Rutkowski, M.D., Winkelstein, B.A., Hickey, W.F., Pahl, J.L., DeLeo, J.A., 2002. Lumbar nerve root injury induces central nervous system neuroimmune activation and neuroinflammation in the rat: relationship to painful radiculopathy. Spine 27, 1604-1613.

Rydevik, B., Hasue, M., Wehling, P., 1996. Etiology of sciatic pain and mechanisms of nerve root compression. In: Wiesel, S.W., Weinsten, J.N., Herkowitz, H., Dvorak, J., Bell, G. (Eds.), The Lumbar Spine. WB Saunders Co., Philadelphia, pp. 123-141.

Saal, J.S., 1995. The role of inflammation in lumbar pain. Spine 20, 1821-1827.

Schiefer, J., Kampe, K., Dodt, H.U., Zieglgansberger, W., Kreutzberg, G.W., 1999. Microglial motility in the rat facial nucleus following peripheral axotomy. J. Neurocytol. 28, 439-453.

Skouen, J.S., Brisby, H., Otani, K., Olmarker, K., Rosengren, L., Rydevik, B., 1999. Protein markers in cerebrospinal fluid in experimental nerve root injury. Spine 24, 2195-2200.

Tabo, E., Jinks, S.L., Eisele Jr., J.H., Carstens, E., 1999. Behavioral manifestations of neuropathic pain and mechanical allodynia, and changes in spinal dorsal horn neurons, following L4-L6 dorsal root constriction in rats. Pain 80, 503-520.

Tanga, F.Y., Raghavendra, V., DeLeo, J.A., 2004. Quantitative real-time RT-PCR assessment of spinal microglial and astrocytic activation markers in a rat model of neuropathic pain. Neurochem. Int. 45, 397-407.

Tsuda, M., Inoue, K., Salter, M.W., 2005. Neuropathic pain and spinal microglia: a big problem from molecules in 'small' glia. Trends Neurosci. 28, 101-107.

Wall, P., Melzak, R., 1994. Textbook of Pain, 3rd edition. Churchill Livingstone, London.

Watkins, L.R., Maier, S.F., 2003. Glia: a novel drug discovery target for clinical pain. Nat. Rev. Drug Discov. 2, 973-985.

Weiler, C., Nerlich, A.G., Bachmeier, B.E., Boos, N., 2004. Expression and distribution of tumor necrosis factor alpha in human lumbar intervertebral discs: a study in surgical specimen and autopsy controls. Spine 30, 44-54.

Winkelstein, B.A., DeLeo, J.A., 2002. Nerve root injury severity differentially modulates spinal glial activation in a rat lumbar radiculopathy model: considerations for persistent pain. Brain Res. 956, 294-301.

Winkelstein, B.A., DeLeo, J.A., 2004. Mechanical thresholds for initiation and persistence of pain following nerve root injury: mechanical and chemical contributions at injury. J. Biomech. Eng. 126, 258-263.

Winkelstein, B.A., Rutkowski, M.D., Sweitzer, S.M., Pahl, J.L., DeLeo, J.A., 2001a. Nerve injury proximal or distal to the DRG induces similar spinal glial activation and selective cytokine expression but differential behavioral responses to pharmacologic treatment. J. Comp. Neurol. 439, 127-139.

Winkelstein, B.A., Rutkowski, M.D., Weinstein, J.N., DeLeo, J.A., 2001b. Quantification of neural tissue injury in a rat radiculopathy model: comparison of local deformation, behavioral outcomes, and spinal cytokine mRNA for two surgeons. J. Neurosci. Methods 11, 49-57. 
Xu, J., Pollack, C.H., Kajander, K.C., 1996. Chromic gut suture reduces calcitonin-gene-related-peptide and substance $\mathrm{P}$ levels in the spinal cord following chronic constriction injury in the rat. Pain 64, 503-509.

Yabuki, S., Kawaguchi, Y., Nordborg, C., Kikuchi, S., Rydevik, B., Olmarker, K., 1998. Effects of lidocaine on nucleus pulposus-induced nerve root injury: a neurophysiologic and histologic study of the pig cauda equina. Spine 23, 2383-2389.

Yamaguchi, K., Murakami, M., Takahashi, K., Moriya, H., Tatsuoka, H., Chiba, T., 1999. Behavioral and morphologic studies of the chronically compressed cauda equina. Spine 24, 845-851.
Yoshida, M., Nakamura, T., Sei, A., Kikuchi, T., Takagi, K., Matsukawa, K., 2005. Intervertebral disc cells produce tumor necrosis factor $\alpha$, interleukin-1 $\beta$, and monocyte chemoattractant protein-1 immediately after herniation: an experimental study using a new hernia model. Spine 30, 55-61.

Zhuang, Z.Y., Gerner, P., Woolf, C.J., Ji, R.R., 2005. ERK is sequentially activated in neurons, microglia, and astrocytes by spinal nerve ligation and contributes to mechanical allodynia in this neuropathic pain model. Pain 114, 149-159.

Zimmermann, M., 1983. Ethical guidelines for investigations of experimental pain in conscious animals. Pain 16, 109-110. 